\title{
EVALUACIÓN DEL HÁBITAT DISPONIBLE PARA DOS ESPECIES DE CÉRVIDOS EN EL NOROESTE DE MÉXICO
}

\author{
MARIO A. SERRA-ORTIZ ${ }^{1}$, FERNANDO N. GONZÁLEZ-SALDIVAR ${ }^{1}$, CESAR \\ CANTÚ-AYALA ${ }^{1}$, JOSE GUEVARA-GONZÁLEZ ${ }^{1}$ Y FRANCISCO PICÓN-RUBIO² \\ ${ }^{1}$ Facultad de Ciencias Forestales, UANL. Carretera Nacional km 145, Linares, \\ Nuevo León, México. \\ ${ }^{2}$ Facultad de Medicina Veterinaria y Zootecnia, UANL. \\ correo electrónico: maserraor@yahoo.com
}

\begin{abstract}
RESUMEN: Se realizó una evaluación del hábitat del venado bura (Odocoileus hemionus eremicus) y venado cola blanca (Odocoileus virginianus couesi), la cual se llevo a cabo en dos diferentes ranchos localizados en el estado de Sonora, México y durante dos épocas del año, la primera durante los meses de octubre, noviembre y diciembre (otoño-invierno), mientras que la segunda se realizó en los meses de junio, julio y agosto (primavera-verano). Las variables evaluadas fueron: la producción de biomasa seca, disponibilidad de agua y cobertura de escape, posteriormente se determino contenido de energía bruta y digestibilidad aparente de la materia seca de las plantas consumidas por los cervidos, a manera de conocer la calidad del alimento consumido por los mismos. Se utilizaron Índices de Disponibilidad (ID) para cada variable para determinar cual de ellos es limitante para el desarrollo de las poblaciones. Se utilizo un modelo mecanistico, en el cual por medio de una media geométrica se integran las tres variables del hábitat, para obtener un Índice de Disponibilidad de Hábitat (IDH). Se evaluaron las poblaciones de ambas especies de cervidos durante las mismas épocas del año, para determinar si la variabilidad entre la calidad del hábitat de los predios es un factor que afecte a estas poblaciones.
\end{abstract}

PALABRAS CLAVE: Índice de disponibilidad, hábitat, Odocoileus, hemionus, virginianus, Sonora, México.

ABSTRACT: We evaluated habitats of mule deer (Odocoileus hemionus eremicus) and white tailed-deer (Odocoileus virginianus couesi) in two different regions "El Plomito" and "El Caracol" in Sonora, México, during two seasons: October-December (fall-winter), and juneaugust (spring-summer). The habitat variables were: biomass production, water availability and escape cover, we determinate gross energy and dry matter digestibility values for each plant consumed by the deer. We utilized Suitability Index (SI) for each variable to determinate which of them are restrictive for the populations of deer's. The food readiness is analyzed giving emphasis in the quality of the food; we use de gross energy values. We utilized a mechanistic model were integrated the habitat variables for determinate a Habitat Suitability Index Model (HIS).

KEY WORDS: Habitat suitability Index, Odocoileus, hemionus, virginianus, Sonora, México. 


\section{INTRODUCCIÓN}

En México se distribuyen cuatro especies de cérvidos, el venado bura (O. hemionus) cuya distribución abarca los estados del norte noroeste del país (Misurata, 1999), el venado temazate (Mazama americana) que se distribuye en las regiones tropicales húmedas, El venado café de Yucatán (Mazama pandora), cuya distribución se limita a los hábitat de la península de Yucatán y el venado cola blanca (O. virginianus) cuya distribución geográfica es la más amplia, encontrándose en una variedad de ecosistemas. La otra subespecie ya extinta el wapiti (Cervus elaphus merriami) se distribuía en los estados del norte del país (Galindo-Leal y Weber, 1998).

La subespecie de venado bura que se distribuye en Sonora es (O.h. eremicus), esta es una de las seis subespecies que se distribuyen en el país. El venado cola blanca de coues (O.v. couesi), se distribuye en la Sierra Madre Occidental abarcando nueve estados de la Republica, en Sonora se le puede encontrar en las sierras del estado, ocupando áreas de matorral desértico micrófilo (Galindo-Leal y Weber, 1998). Estas dos subespecies habitan en simpatría dentro del área de estudio y se encuentran sujetas a las mismas presiones como lo son la ganadería, degradación y pérdida del hábitat y la cacería controlada.

Una de las herramientas utilizada por los ganaderos o manejadores de fauna silvestre es el manejo o mejoramiento del hábitat, lo cual generalmente se lleva a cabo sin la ayuda de herramientas que ayuden a la mejor toma de decisiones.

Existen trabajos donde se evalúa y valora el hábitat del venado cola blanca tejano principalmente (O.v. texanus), uno de los cuales el de Short en 1986, desarrollada un Modelo de Índice de Disponibilidad de Hábitat para esta especie, en las costas del Golfo de México y las planicies costeras del Atlántico sur. En México los trabajos que se han desarrollado son principalmente para el cola blanca (O. virginianus), siendo la subespecie tejana la más estudiada, dado que es la que mayor valor cinegético tiene en el país, mientras que el venado de coues en la segunda subespecie mas estudiada (Mandujano, 2004).

El presente trabajo utiliza como guía el trabajo desarrollado por Short (1986), el cual se adapto a las condiciones existentes en Sonora, con la finalidad de conocer las condiciones actuales del hábitat y determinar mediante un Índice de Disponibilidad de Hábitat (IDH), cual o cuales de la variables del hábitat limitan el desarrollo natural de las poblaciones de ambas especies.

\section{ÁREADEESTUDIO}

Se trabajó en dos predios ubicados en el Municipio de Pitiquito, en el estado de Sonora, México. El primero llamado "El Plomito", localizado en la porción sur del complejo Sierra del Viejo, dentro de la región terrestre prioritaria 16 Sierra El Álamo-El 
Viejo a $70 \mathrm{Km}$. de la ciudad de Caborca, abarca una superficie de 10, 375 has constituidas principalmente de lomeríos y llanuras. El segundo rancho llamado "El Caracol" con una superficie aproximada de 4,500 has, localizado cerca de la sierra El Caracol a $20 \mathrm{Km}$. de Puerto Libertad.

El clima según la clasificación climática de Köppen modificada por García (1998) es del tipo BWh(x') descrito como muy árido, semicalido, lluvias entre verano e invierno mayores a $18 \%$ anual. La temperatura media anual es de $22.2^{\circ} \mathrm{C}$, siendo julio el mes más cálido con un promedio de $32.0^{\circ} \mathrm{C}$ y el más frió Diciembre con $12.6^{\circ} \mathrm{C}$. La precipitación media anual es de $266.5 \mathrm{~mm}$, los meses con menor precipitación son mayo y junio con 1.3 y $0.8 \mathrm{~mm}$ y los meses con mayor precipitación son julio y agosto con 68.4 y $66.3 \mathrm{~mm}$ (INEGI, 2008).

Los tipos de vegetación presentes en el área de estudio según la clasificación de INEGI (1997) son: Matorral Sarcocaule y Matorral Desértico Micrófilo. Entre las especies más comunes en el área de estudio encontramos el palo verde (Cercidium microphyllum), palo fierro (Olneya tesota), mezquite (Prosopis glandulosa), sahuaro (Carnegeia gigantea), jojoba (Simmondsia chinensis), torote prieto (Bursera microphylla). (Hernández, 1998; Olguín, 2001).

\section{MÉTODOS}

El trabajo se dividió en dos épocas del año las cuales se consideran como criticas para estas especies, la primera en el otoño, en los meses de octubre-diciembre de 2004, la segunda etapa en el verano, la cual comprendió los meses de junio-agosto del 2005. Se evaluaron en campo tres variables del hábitat de estas especies, la disponibilidad de alimento (biomasa seca), agua y cobertura horizontal de la vegetación (cobertura de escape). Posterior a esto en el Laboratorio de Nutrición Animal de la Facultad de Medicina Veterinaria y Zootecnia de la UANL se llevaron acabo análisis de contenido de energía bruta y digestibilidad aparente de la materia seca, para cuantificar de está manera la calidad del alimento consumido por los venados. Así mismo se realizó una caracterización del hábitat para conocer las especies que dominan el paisaje del área de estudio.

\section{Caracterización del hábitat}

Se utilizó el método de la línea de intercepto o de Canfield, el cual se emplea para determinar cobertura y frecuencia de especies. (Bautista et al., 2004; Hays, 1981). Se utilizaron las siguientes formulas para el calculó de Cobertura, Frecuencia y Dominancia:

Cobertura (C):

$$
C=\frac{L}{L t} \times 100
$$


Donde:

$\mathrm{L}=$ Longitud interceptada por especie.

$\mathrm{Lt}$ = Longitud total de las líneas.

Frecuencia (F):

$$
\mathrm{F}=\frac{\mathrm{Ni}}{\mathrm{Ni}} \times 100
$$

Donde:

$\mathrm{Ni}=$ Número de veces que la especie es interceptada.

$\mathrm{Nt}=$ Total de especies interceptadas

Dominancia (D):

$$
\mathrm{D}=\frac{\sum \text { intercepto de } \mathrm{xi}}{\sum \text { intercepto total }} * 100
$$

Donde:

$\sum x i=$ Sumatoria de los interceptos de la especie $i$ en una línea de muestreo

$\sum$ total $=$ Sumatoria total de todos los interceptos de todas las especies

\section{Índice de disponibilidad de hábitat}

\section{Producción de biomasa}

Para evaluar la producción de biomasa seca se dividió la vegetación en tres estratos, el estrato bajo, menor de 0.3 metros, estrato medio que va de 0.3 a 1.5 metros y el estrato alto mayor de 1.5 metros de altura. Para el estrato medio y alto se utilizó el método de Adelaide o unidad de referencia de mano y para el estrato bajo el método de parcela de corte (González, 1996).

\section{Disponibilidad de agua}

La disponibilidad de agua se determinó ubicando en campo las fuentes de agua permanentes las cuales se georreferencian. Estas fuentes de agua fueron ubicadas y desplegadas en un mapa digital utilizando el programa Arcview versión 3.2, a cada fuente de agua se le colocó un buffer de 800 metros de radio, se asume que la distancia entre fuentes de agua debe ser de $1.6 \mathrm{Km}$., ya que usualmente el área de actividad de un venado cola blanca no excede de $2.6 \mathrm{~km}^{2}$ [Observaciones de Severinghaus y Cheatum, (1956) citado por Short (1986)]. 


\section{Cobertura de escape}

La cobertura de escape se evaluó utilizando un telémetro (rangefinder). Dicha medición consistió en medir la distancia a la cual un objeto en movimiento rectilíneo se pierde de vista a una altura de 1.4 metros, esta pérdida de vista del objeto se debe principalmente a la densidad de la vegetación presente, áreas con mayor vegetación presenta una menor distancia de perdida de vista y vegetación con menor densidad presenta una mayor distancia para la pérdida de vista.

\section{Contenido de energía y digestibilidad}

Se colectaron muestras de todas las plantas presentes en el área de estudio, durante ambas épocas del año, las cuales fueron puestas en bolsas de papel y secadas en estufa de flujo de aire, fueron identificadas para posteriormente ser molidas utilizando primeramente un molino Retsch Mühle con criba de $2 \mathrm{~mm}$, posteriormente se utilizo un molino Thomas Scientific modelo 3379-L10 con criba \# 40. A estas muestras se les determino el contenido de energía bruta y digestibilidad aparente.

\section{Energía bruta}

La energía bruta se determinó utilizando el método de la bomba calorimétrica (Galyean, 1997; Shimada, 2003). Se utilizo la siguiente formula para el calculo del contenido de energía bruta.

EB = (Temp Inicial - Temp Final) 2412-(cm alambre x calorias del alambre) ml Titulación grs muestra

Donde:

$E B$ = Contenido de energía bruta de la muestra

Temp Inicial = temperatura inicial del agua que rodea la bomba calorimétrica

Temp Final = temperatura final del agua que rodea la bomba calorimétrica 2,412 = constante de la bomba calorimétrica cm alambre = los centímetros de fusible consumidos por la combustión calorías del alambre = constante de la marca del fusible ml titulación = mililitros de carbonato de sodio monohidratado utilizados grs muestra $=$ peso seco del pelet de muestra utilizado 


\section{Digestibilidad aparente}

La digestibilidad aparente se determinó utilizando el método in situ de la bolsa de nylon (Ramírez, 2003). Se utilizaron cuatro borregos fistulado, con una alimentación controlada dos semanas antes del inicio del experimento, el tiempo de incubación utilizado fue de 48 horas. Se hace la mención que para la utilización del modelo disponibilidad de hábitat, solo se tomaron en cuenta las plantas que son consumidas por ambas especies y las cuales aparecen en la lista de especies que forman su dieta.

\section{Modelo de índice de disponibilidad de hábitat (IDH)}

Se elaboro este modelo teniendo como guía el desarrollado por Short (1986), el cual fue descrito para ser utilizado en las costas del golfo en los EUA. En este caso fue adaptado para su aplicación en el desierto sonorense, en el noroeste de México, específicamente en el estado de Sonora. La vegetación presente en el área es del tipo matorral sarcocaule y matorral desértico micrófilo (Figura 1).

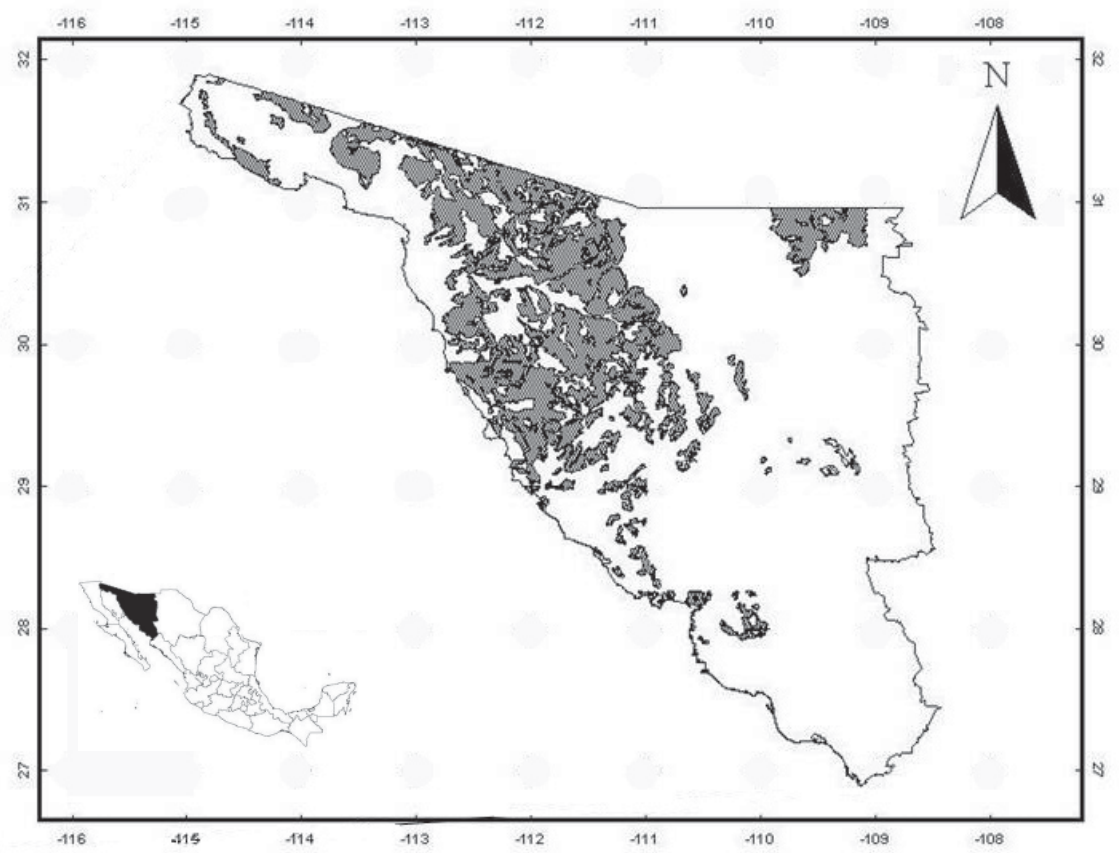

Figura 1. Modelo del índice de disponibilidad del hábitat de cérvidos en Sonora, México. 
El IDH indica mediante un valor numérico entre 0-1 la calidad del hábitat evaluado, 0 indica un hábitat nulo, mientras que 1 indica un hábitat optimo. Así mismo, brinda información importante de la o las variables del hábitat limitantes para el correcto desarrollo de las poblaciones de fauna silvestre y en este caso en específico, de los cervidos presentes en el área.

Este modelo se basa principalmente en la cantidad y calidad de alimento disponible para los cervidos en forma de energía metabolizable y la disponibilidad del agua en el área, estas dos variables se determinaron como las de mayor importancia para las especies en el área de estudio. La cantidad de EM presente en el área de estudio se compara con la EM presente dentro de unidades estándar de hábitat, la cual se define como la energía necesaria para que un venado lleve a cabo sus actividades fisiológicas durante 42 días, contenida en una hectárea de hábitat (Short, 1986).

Se elaboró un modelo matemático mecanistico mediante una media geométrica, en el cual se integran las variables del hábitat evaluadas en campo y laboratorio (Brockington, 1979; Muniz, 2007; Silva, 1993).

Se utilizó la siguiente formula para el cálculo de IDH (Modelo Mecanistico):

IDH Total $=\frac{(\text { IDE * IDA })^{1 / 2}+\text { IDC }}{2}=$ Valor de Índice de Disponibilidad de Hábitat

Donde:

$I D E$ = Índice de disponibilidad de energía

IDA = Índice de disponibilidad de agua

$I D C=$ Índice de disponibilidad de cobertura de escape

Para el cálculo de cada uno de los índices de disponibilidad se utilizaron las siguientes formulas:

$$
\mathrm{IDE}=\frac{\sum_{\mathrm{i}=1}^{\overline{\mathrm{n}}}(\mathrm{CFi} * \mathrm{DFi} * \mathrm{VEi})}{\text { Kcal EM ha hábitat óptimo }}
$$

Donde:

$\mathrm{n}$

$\Sigma$ = Cada una de las especies vegetales consumidas por los cervidos presentes en el $\mathrm{i}=1$ área de estudio expresado en grs/ha.

$C F=$ La cantidad en gramos de cada especie vegetal disponible como alimento para los venados (las que son consumidas por la especie).

$D F=$ La digestibilidad aparente de cada especie vegetal consumida por los venados durante la época de verano.

$V E=E l$ valor de energía de la especie consumida, la cual es igual al valor de energía bruta (EB) multiplicada por una constante 0.8 , lo que nos provee un estimado de la 
energía metabolizable (EM) después de que la digestibilidad del forraje ha sido determinada.

kcal EM/ha de hábitat optimo = La cantidad de energía metabolizable que esta disponible para los venados en unidades estándar de hábitat.

$$
\text { IDA }=\frac{\text { \# fuentes de agua } * \text { cobertura efectiva has }}{\text { superficie predio (has) }}
$$

$I D A=$ Índice de disponibilidad de agua

\# Fuentes agua = las fuentes de agua permanentes en el predio

Cobertura efectiva = superficie en hectáreas que cubre una fuente de agua

$$
\text { IDC }(\text { escape })=\frac{\text { distancia medida }(\mathrm{m})}{50 \mathrm{~m}}
$$

IDC (escape) = Índice de disponibilidad de cobertura de escape

Distancia medida =distancia medida en metros de cobertura de escape

$50 \mathrm{~m}$ = valor optimo de cobertura de escape

\section{Densidades poblacionales de cervidos}

Se utilizó el método de conteo de excretas; se utilizaron transectos de un kilómetro de largo, con una parcela cada 100 metros de $10 \mathrm{~m}^{2}$ cada una, las cuales son limpiadas de excretas viejas, pasando 40 días, se revisan y se cuentan los grupos de excretas presentes.

Para el cálculo de las poblaciones de venados por medio del método de conteo de excretas se utiliza la siguiente ecuación (Eberhardt y Van Etten, 1956; González, 1996; Pérez-Mejía, 2004):

$$
\text { Venados } / \mathrm{Km}^{2}=\frac{\mathrm{PG} * \mathrm{NP}}{\mathrm{TP} * \mathrm{TD}}
$$

Donde:

$P G=$ Media de grupos de excretas

$N P=$ Número de parcelas

$T P=$ Tiempo de deposición de excretas

$T D=$ Tasa de defecación diaria utilizada (12.7) 


\section{RESULTADOS}

\section{Caracterización del hábitat}

Se encontraron variaciones en los valores de cobertura vegetal, en "El Plomito", la cobertura en la época de otoño fue de 20.7 \%, mientras que para la época de verano el valor de cobertura fue de $29.5 \%$; en "El Caracol", los valores de cobertura fueron similares, encontrando un $35.9 \%$ de cobertura en el otoño y $37.8 \%$ de cobertura en el verano. Las especies que presentan mayor frecuencia dentro del área de estudio son: (Euphorbia polycarpa con $16.1 \%$, (Jathropha cuneata) 15.7 \% y (Encelia farinosa) 9.5 \% esto para el área de "El Plomito", mientras que para el área de "El Caracol", las especies que con mayor frecuencia se encuentran son (J. Cuneata) $8.1 \%$, (E. Farinosa) $7.5 \%$ y (Cryptantha maritima) 7.7\%. En cuanto a la dominancia las especies que presentaron valores más altos tenemos: (J. cuneata) con $16.3 \%$, (Larrea tridentata) con $12.4 \%$ y (Cercidium microphyllum) $12.3 \%$. Mientras que en "El Caracol" se encontró a (J. Cuneata) 12.9 \%, (L. tridentata) 8.4 \% y (Bursera microphylla) $6.8 \%$.

\section{Índice de disponibilidad de hábitat}

\section{Producción de biomasa seca}

En cuanto a la producción de biomasa tenemos que el verano (junio-agosto) fue la época en que menor biomasa seca total se produjo en ambos predios, con valores de: $589 \pm 20.2$ kg/ha de materia seca en "El Plomito" y en "El Caracol", una producción de biomasa de $256.5 \pm 11.6 \mathrm{~kg} / \mathrm{ha}$ de materia seca, de esta biomasa solo la cuarta parte se considero como disponible como alimento para los venados (Figura 2).

\section{Contenido de energía bruta}

El contenido de energía bruta (EB) expresado en kilocalorías por gramo de muestra (Kcal/gr), para la época de otoño, fue en promedio de $4.37 \mathrm{Kcal} / \mathrm{gr}$ mientras que para el verano, fue de $3.94 \mathrm{Kcal} / \mathrm{gr}$. Las especies que presentaron los valores de energía mas altos durante la época de otoño fueron: (Hyptis albida) con 8.7 y (Krameria erecta) $6.4 \mathrm{Kcal} / \mathrm{gr}$, mientras que para el verano fueron: (Simmondsia chinensis) (fruto) $5.7 \mathrm{y}$ (Cercidium microphyllum) (vainas) $5.3 \mathrm{kcal} / \mathrm{gr}$.

\section{Digestibilidad aparente de la materia seca}

Los porcentajes de digestibilidad por especie variaron grandemente durante la misma estación del año, siendo en el otoño el más bajo, (K. erecta) con 6.4 \% y (Cordia 


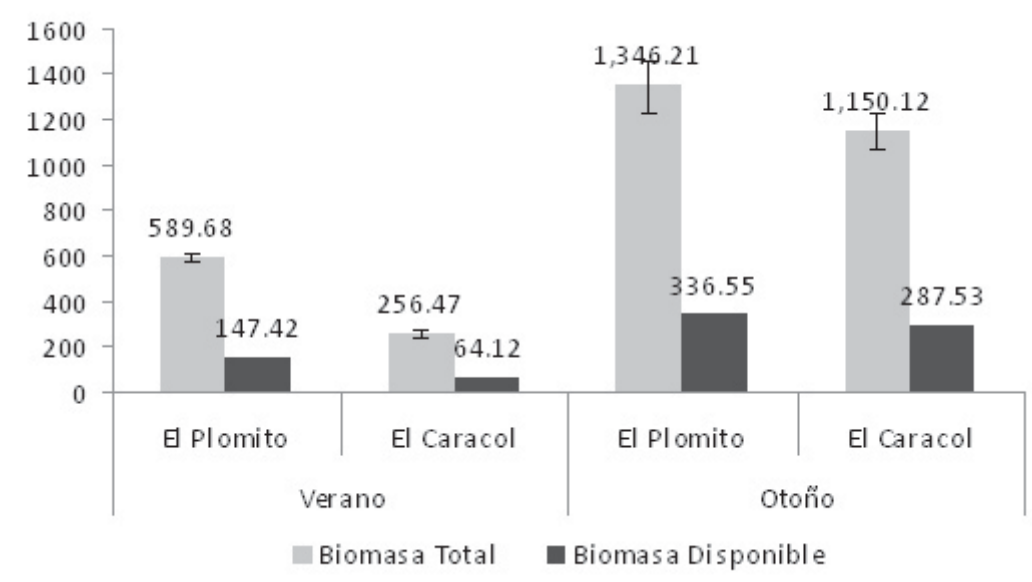

Figura 2. Producción de Biomasa Seca en el Área de Estudio.

parvifolia) con $7.8 \%$ de Digestibilidad. Mientras que las especies que presenta mayor digestibilidad son: (Opuntia leptocaulis) con $50.0 \%$ y (Celtis pallida) con $49.9 \%$ de digestibilidad.

En cuanto a la época de verano, las especies que presenta menor valor de digestibilidad son: (H. albida) con 4.3 \% y (Descurainia pinnata) con $10.8 \%$ de digestibilidad. Por otro lado las especies que presentan mayores porcentajes de digestibilidad son: (Carnegiea gigantea) 63.4 \% y (Havardia sonorae) (vaina) con $50.2 \%$ de digestibilidad.

\section{Cobertura de escape}

Se encontró un tipo de vegetación: el matorral desértico micrófilo, con dos asociaciones vegetales Larrea-Franseria y Larrea-Simmondsia, en el área de "El Plomito", mientras que para "El Caracol" se clasificó únicamente como matorral desértico micrófilo. La época de otoño presentó mejores valores promedio en las tres asociaciones siendo la mejor la asociación Larrea-Simmondsia con un valor de 39.3 metros (Figura 3).

\section{Disponibilidad de agua}

Las fuentes de agua permanentes que se localizaron en el área de estudio fueron para "El Plomito" una red de 11 bebederos, mientras que en "El Caracol" el agua se 
distribuye en una red de 4 bebederos (Figura 4). La cobertura efectiva de las fuentes de agua en "El Plomito" es del 49 \% de la superficie, mientras que en "El Caracol" es del 17\% de la superficie. Cabe hacer mención que en "El Plomito", la red de bebederos durante el verano no funciono debido a la descompostura de la bomba de agua que provee del líquido a la misma.

\section{Calculo de los índices de disponibilidad}

La energía disponible como alimento para los venados durante la época del verano se calculó en base a la dieta de cada una de estas especies de venado, los resultados muestran una menor disponibilidad de alimento en "El Caracol" (Cuadro 1).

Analizando independientemente las variables del hábitat se encontró que la disponibilidad de agua es la variable con el índice de hábitat más bajo, el cual es de 0.5 en "El Plomito" y 0.2 en "El Caracol". Mientras que la cobertura de escape obtuvo los valores de índice más altos, siendo este de 1 en "El Plomito" y 0.9 en "El Caracol" (Cuadro 2).

El calculó del IDH muestra que existen mejores condiciones de hábitat en "El Plomito" con un valor de 0.7 para el bura y 0.8 para el cola blanca.

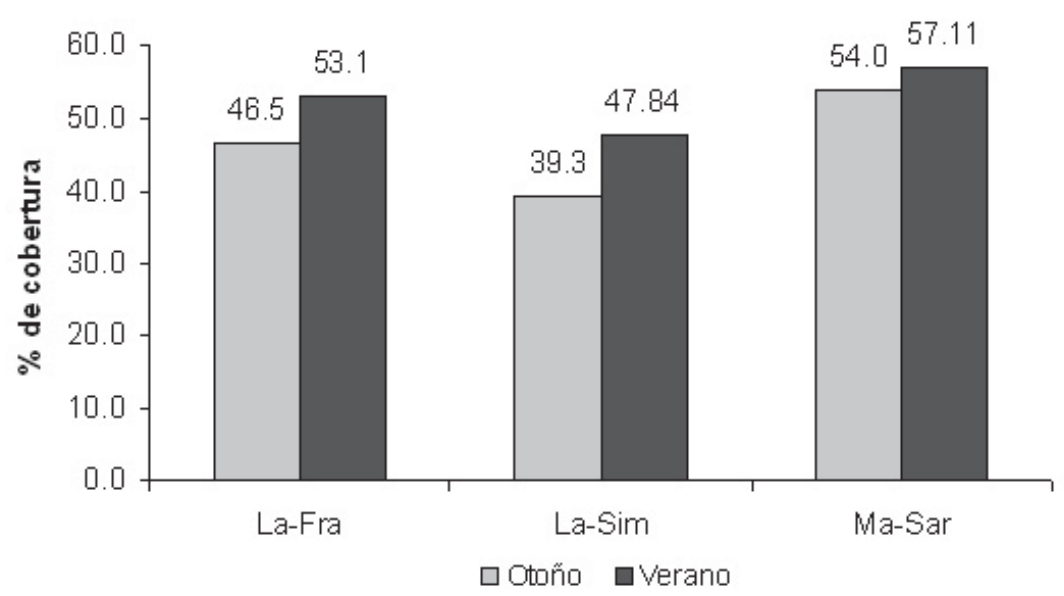

Figura 3. Valores de cobertura de escape en el área de estudio, en ambas épocas del año. 


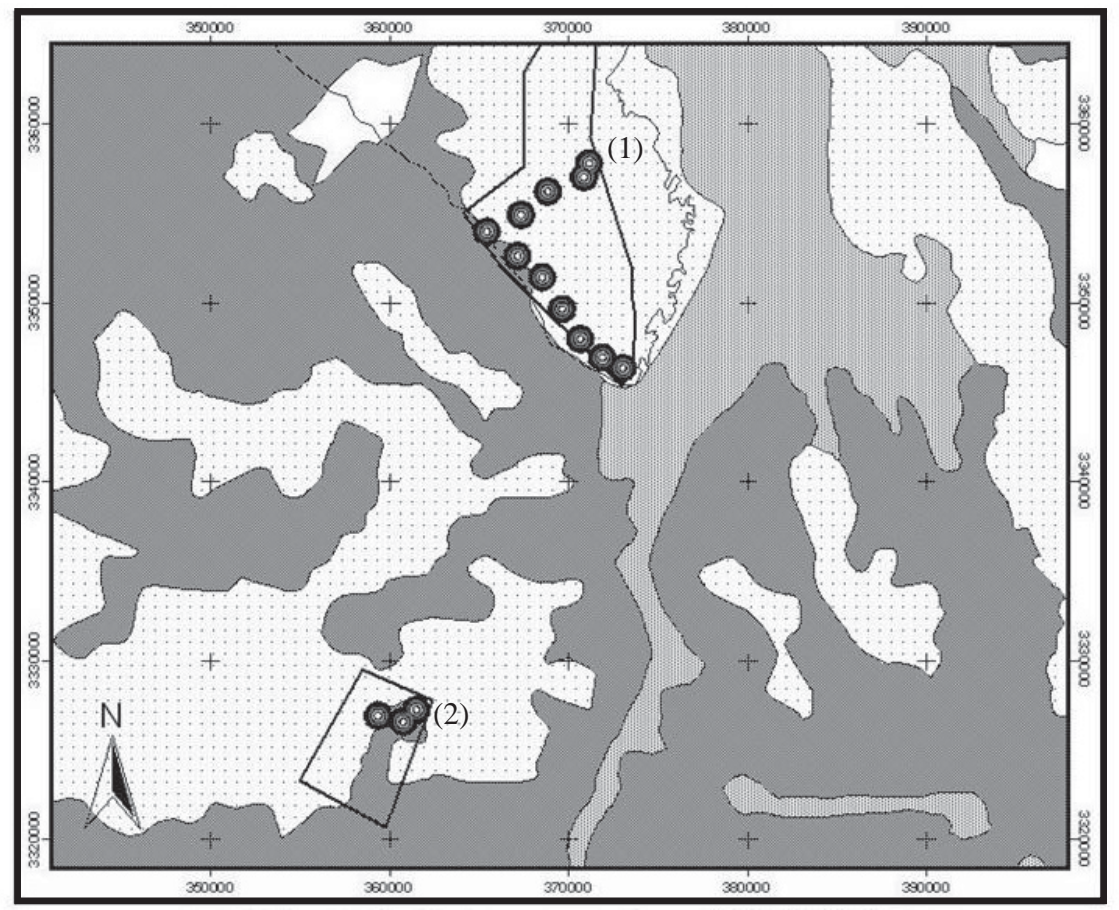

Figura 4. Fuentes de agua permanente y su cobertura efectiva en "El caracol" (1) y "El Plomito" (2). Los cuatro tipos de cobertura se indican en tonos de grises: (blanco)200, (gris claro)400, (gris oscuro) 600 y (negro) 800, y las fuentes de agua con un punto blanco.

Cuadro 1. Energía disponible en el área de estudio para cada especie de venado.

\begin{tabular}{llll}
\hline & $\begin{array}{l}\text { Kcal /EM/ha } \\
\text { Hábitat óptimo }\end{array}$ & $\begin{array}{l}\text { Kcal/EM /ha } \\
\text { Caracol }\end{array}$ & $\begin{array}{l}\text { Kcal/ EM /ha } \\
\text { Plomito }\end{array}$ \\
\hline Venado Bura & 135,403 & 34,196 & 69,404 \\
Venado Cola Blanca & 99,172 & 25,555 & 60,256 \\
\hline
\end{tabular}

Kcal/EM/ha/hábitat óptimo = la energía que habría en una hectárea de hábitat óptimo para la especie durante un periodo de 45 días.

$\mathrm{Kcal} / \mathrm{EM} / \mathrm{ha}=\mathrm{PM} * \mathrm{RAM} * 70$

$\mathrm{PM}=$ peso metabólico de un venado

RAM = rango de actividad metabólica

$70=$ constante 
Cuadro 2. Índices de disponibilidad determinados para cada variable del hábitat evaluada.

\begin{tabular}{|c|c|c|c|c|c|}
\hline Predio & Especie & IDE & IDA & IDC & IDH \\
\hline \multirow[t]{2}{*}{ "El Plomito" } & Odocoileus hemionus & 0.5 & 0.5 & 1 & 0.7 \\
\hline & Odocoileus virginianus & 0.6 & 0.5 & 1 & 0.8 \\
\hline \multirow[t]{2}{*}{ "El Caracol" } & Odocoileus hemionus & 0.3 & 0.2 & 0.9 & 0.5 \\
\hline & Odocoileus virginianus & 0.3 & 0.2 & 0.9 & 0.5 \\
\hline \multicolumn{6}{|c|}{ IDE = índice de disponibilidad de energía } \\
\hline \multicolumn{6}{|c|}{ IDA = índice de disponibilidad de agua } \\
\hline \multicolumn{6}{|c|}{ IDC = índice de disponibilidad de cobertura de escape } \\
\hline \multicolumn{6}{|c|}{ IDH = Índice de Disponibilidad de Hábitat } \\
\hline
\end{tabular}

\section{Densidades poblacionales de cervidos}

Se registraron en "El Plomito" en la época de otoño-invierno densidades poblacionales de 2.3 y 0.18 venados por $\mathrm{km}^{2}$ respectivamente; mientras que para primavera-verano las densidades encontradas fueron de 0.5 venados por $\mathrm{km}^{2}$ para cada una de las especies de venado presente en el área.

En "El Caracol" las densidades encontradas en otoño-invierno fueron de 1.8 y 0.5 venados por $\mathrm{km}^{2}$ para cada una de las especies de venado. Para la época del verano las densidades encontradas de 2.5 y 0.5 venados por $\mathrm{km}^{2}$, lo que significa que mientras que la población de $O$. virginianus prácticamente se mantuvo estable, la población de $O$. hemionus aumentó en un $36.8 \%$.

Las densidades poblacionales de venado bura son similares a las encontradas por Lozano ${ }^{23}$, quien reporto una densidad de 2.2 venados $/ \mathrm{km}^{2}$ de venado bura en el noreste de Coahuila y 5 venados bura por $\mathrm{km}^{2}$ en un área de nuevo México (Figura 5).

\section{DISCUSIÓN}

El IDH nos indica una mejor calidad de hábitat en "El Plomito" esto se debe principalmente a la mayor disponibilidad de agua en el predio, lo cual se logra en base a la red de bebederos que existe en dicho predio, si bien es cierto que los venados obtienen agua de otras fuetes de agua como lo son las flores y frutos de temporada (Villareal Espino-Barros y Marin-Fuentes, 2005), son necesarias estas fuentes de agua para cubrir los requerimientos de agua, la cual para el venado cola blanca es de entre 2 a 4 litros por día (Alcalá y Enríquez, 1999). 


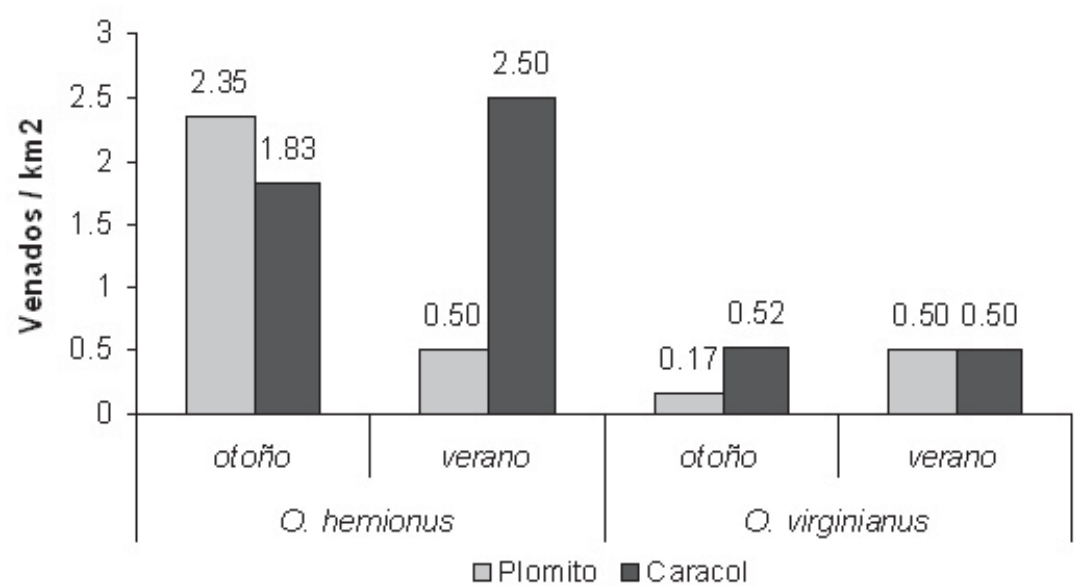

Figura 5. Densidades poblacionales de cervidos.

Observando los valores de energía y digestibilidad de las plantas disponibles como alimento para estos cervidos, podemos observar que en ambas épocas del año los valores promedio se encuentran por debajo de los requerimientos para estas especies, ya que para cubrir las necesidades metabólicas y de lactancia es necesario que consuma especies con contenidos de energía entre 4.3 y $4.8 \mathrm{kcal} / \mathrm{gr}$ (Short, 1986) y una digestibilidad entre 55-60 \% (Varner y Hudges, 1981).

La cobertura vegetal horizontal de la vegetación (cobertura de escape) no es una limitante del hábitat para estas especies de cervidos ya que se cuenta con valores promedio de 49.5 metros, lo cual se puede considerar como optimo para estas especies (Chávez, 1994; Short, 1986).

El IDH muestra una mejor calidad de hábitat en "El Plomito" con un valor de 0.7 y 0.8 para bura y cola blanca respectivamente. Este valor de IDH es similar al determinado por Chávez (1994), en el cual la disponibilidad de agua es la variable con valor de índice más bajo.

\section{CONCLUSIONES}

En base a los datos arrojados por el modelo se observa que el factor del hábitat que limita el desarrollo de las poblaciones de cervidos en el área es el agua, se observa esto con la drástica disminución de las poblaciones de ambas especies en El Plomito 
durante el verano, predio que sin embargo muestra los mejores valores en cuanto a la calidad del alimento que estas especies consumen.

\section{AGRADECIMIENTOS}

A la Facultad de Ciencias Forestales de la UANL (Proyecto PAICYT 2005), la Organización Vida Silvestre A.C. (OVIS A.C.) y la Facultad de Medicina Veterinaria y Zootecnia (Laboratorio de Nutrición Animal), por el apoyo económico y las instalaciones utilizadas durante el desarrollo de esta investigación.

\section{LITERATURACITADA}

Alcalá, C. y E. Enríquez. 1999. Manejo y Aprovechamiento de Venados. INIFAP (Instituto Nacional de Investigaciones Forestales, Agrícolas y Pecuarias); Centro de Investigación Regional de Noroeste; Campo Experimental Carbó. Folleto Técnico No. 3.

Bautista, F.Z., H.G., Delfín, J.L., P. Palacio y M.C. Delgado. 2004. Técnicas de Muestreo para Manejadores de Recursos Naturales. Editado por La Dirección General de Estudios de Posgrado, UNAM. INE. México.

Chávez, O.G. 1994. Evaluación del hábitat y determinación de un índice de disponibilidad de hábitat para venado cola blanca texano (Odocoileus virginianus texanus) en Anáhuac, Nuevo León. Tesis de Licenciatura. Facultad de Ciencias Forestales. U.A.N.L., Linares Nuevo León, México.

Galyean, M.L., 1997. Laboratory Procedures in Animal Nutrition Research. $12^{\text {th }}$ edition Texas Tech University. USA.

Eberhardt, L. y R. Van Etten 1956. Evaluation of de Pellet Group Count as a Deer Census Method. Journal of Wildlife Management, 20:70-74.

Galindo-Leal, C. y M. Weber. 1998. El venado de la Sierra Madre Occidental: ecología, manejo y conservación. EDICUSA-CONABIO.

García, E. 1998. Climas: Clasificación de Köppen. Escala 1:1 000000.

González, F.N. 1996. Evaluación de recursos forestales: Evaluación del recurso fauna silvestre. Para nivel de postgrado, Facultad de Ciencias Forestales, UANL, México.

Hays, W.L. 1981. Statistics. 3rd ed. New York: Holt, Rinehart and Winston.

Hernández, C.M.C. 1998. Caracterización de la vegetación del predio "El Plomito", Municipio de Pitiquito, Sonora, México. Tesis Profesional, Universidad Autónoma de Nuevo León.

INEGI. 1997. Base de Datos Geográficos. Diccionario de Datos de Uso de Suelo y Vegetación. (vectorial) escala 1:250,000.

INEGI. 2008. Sistemas Nacionales Estadísticos y de Información Geográfica. Sitio del INEGI en Internet www.inegi.gob.mx

Mandujano, S. 2004. Análisis bibliográfico de los estudios de venados en México. Acta Zoológica Mexicana (nueva serie), 20:211-251.

Misuraca, M. 1999. Odocoileus hemionus (On-line), Animal Diversity Web. Disponible en: http://animaldiverity.ummz.umich.edu/site/accounts/information/Odocoileus_hemionus.html 
Muniz, L.C., M.A.O., Viu, C.U., Magnabosco y D.L.,Toledo. 2007. Modelagem e simulação na agropecuária. PUBVET, Londrina, V. 1, N. 11, Dez 3. Disponible en: http:// www.pubvet.com.br/texto.php?id=106.

Olguín, C.A. 2001. Uso de fuentes de agua por el borrego cimarrón en el rancho "El Plomito", Municipio de Pitiquito, Sonora México. Tesis de Licenciatura. Chapingo, México.

Pérez-Mejía, S., S. Mandujano y L.E., Martínez-Romero. 2004.Tasa de defecación del venado cola blanca, Odocoileus virginianus mexicanus, en cautividad en puebla, México. Acta Zoológica Mexicana (n.s.), 2:167-170.

Ramírez, R.G. 2003. Nutrición de Rumiantes, Sistemas Extensivos. Editorial Trillas, México. Shimada, M.A. 2003. Nutrición Animal. Editorial Trillas, México.

Severinghaus, C.W. y E.L. Cheatum. 1956. The life and times of the white-tailed deer. Pp. 57186, en: The deer of North America Stackpole Books. (W.P., Taylor, ed.) Harrisburg, Pennsylvania.

Silva, M. 1993. Modelos y su Uso en Producción Animal. en: Análisis de Sistemas en Producción Animal: Teorías y Aplicaciones. (M., Silva y A. Mansilla, eds.). Facultad de Ciencias Agrarias y Forestales, Universidad de Chile, en línea:

http://mazinger.sisib.uchile.cl/repositorio/lb/ciencias_agronomicas/silvam01/

Short, H.L. 1986. Habitat suitability index models: White-tailed deer in the Gulf of México and South Atlantic coastal plains. U.S. Fish Wildlife Service Biology Rep. 82(10.123).

Uvalle, J. 1998. Evaluación del Hábitat y de las Poblaciones del Venado Bura del Desierto (Odocoileus hemionus crooki) en la Región Cinegética tres del Estado de Coahuila. Tesis de Licenciatura, Facultad de Ciencias Forestales, UANL.

Varner, L.W y H.G, Hudges. 1981. Nutrition Effects on Fawn, Doe and Buck Deer. Proc. International Ranchers Roundup. Texas Ag. Extension Service, Del Rio, Texas. http:// wildlife.tamu.edu/publications.cfm?catID $=6$

Villarreal-Espino, O. y M. Marín-Fuentes. 2005. Agua de origen vegetal para el venado cola blanca mexicano. Archivos Zootécnicos, 54:191-196. 